\title{
Characteristics of patients with novel coronavirus disease (COVID-19) during the first surge versus the second surge of infections in Osaka Prefecture, Japan
}

\author{
Taro Takeuchi ${ }^{1}$, Tetsuhisa Kitamura ${ }^{1, *}$, Atsushi Hirayama ${ }^{2}$, Yusuke Katayama ${ }^{3}$, Takeshi Shimazu ${ }^{3}$, Tomotaka Sobue \\ ${ }^{1}$ Department of Social Medicine, Osaka University Graduate School of Medicine, Suita, Japan; \\ ${ }^{2}$ Department of Public Health, Osaka University Graduate School of Medicine, Suita, Japan; \\ ${ }^{3}$ Department of Traumatology and Acute Critical Medicine, Osaka University Graduate School of Medicine, Suita, Japan.
}

\begin{abstract}
In Japan, the differences in characteristics, severity, and mortality of novel coronavirus disease (COVID-19) patients between the first and second surges of infections have not been fully understood. This study is a retrospective cohort study of COVID-19 patients confirmed between February 1 and August 31, 2020 in Osaka Prefecture, Japan. Publicly available information on patients was collected from the website of Osaka Prefecture. Patients were divided into two groups according to the date of the positive laboratory test result: the first surge (February 1 to May 22) and the second surge (May 23 to August 31). Patients' characteristics were compared between the two groups. A multivariable Cox proportional-hazards model was applied to compare severity and mortality between the two groups, where sex, age group at the onset date, city of residence, and days to test positive were adjusted. Hazard ratios (HRs) with $95 \%$ confidence intervals (CIs) were calculated. A total of 8,541 patients included 1,780 and 6,761 patients in the first and second surges, respectively. Age at the onset date was younger in the second surge $(p<0.001)$, and median of days from the onset date to the positive test date shortened from 7 to 6 days $(p<0.001)$. The multivariable Cox proportionalhazards model revealed that both severity and mortality were lower in the second surge than in the first surge (severity: HR: 0.51 [0.39-0.67]; mortality: HR: 0.37 [0.25-0.56]). In conclusion, severity and mortality were lower in the second surge than in the first surge among COVID-19 patients in Osaka Prefecture, Japan.
\end{abstract}

Keywords: SARS-CoV-2, severity, mortality, survival analysis

\section{Introduction}

The novel coronavirus disease 2019 (COVID-19), which was identified in Wuhan, China in December 2019, has spread to countries all over the world (1-3). In Japan, the cumulative number of patients was 67,865 on August 31,2020 (4). The daily number of newly diagnosed patients has changed over time. The first COVID-19 case in Japan was identified on January 16, 2020, and subsequently, the daily number of patients began to rise (the first surge), and reached its first peak on April 10,2020 (4). Although the daily number of patients decreased after the initial peak, it started to increase again since the middle of June (the second surge), and reached its second peak on August 7, 2020 (4). Although the daily number of patients decreased after the second peak, it started to increase again in October. As of December 2020, Japan is in the middle of the third surge of infections (4). Although a prior study (5) compared the characteristics of COVID-19 patients between the first and second surges in the United States, little is known about the differences in characteristics, severity, and mortality of COVID-19 patients between the first and second surges.

Osaka Prefecture is the largest metropolitan area in the western part of Japan, with a population of 8.8 million, and a total area of $1,905 \mathrm{~km}^{2}(6)$. It has the second highest number of COVID-19 patients in Japan next to Tokyo $(4,6)$. The present study aimed to assess the difference in characteristics, severity, and mortality of COVID-19 patients between the first and the second surges of infections in Osaka Prefecture, Japan.

\section{Methods}

Study design

This study was a retrospective observational study conducted from February 1 to August 31, 2020. Publicly available data on laboratory-confirmed COVID-19 patients in Osaka Prefecture, Japan, were collected from the Osaka Prefecture website (7). Since we only collected anonymous data, the necessity of obtaining informed consent from the participants was waived (6). 
This study was approved by the Ethics Committee of Osaka University Graduate School of Medicine (approval No. 20089).

Official data collection of COVID-19 patients in Osaka Prefecture

Details on the data collection have been previously published elsewhere (6) and are available on the Osaka Prefecture website (7). In brief, the following information on COVID-19 patients is available on the website of Osaka Prefecture: sex, age group at the onset date, city of residence, onset date, date when the patient showed positive results in the laboratory test, date when a patient's condition became severe (for patients whose condition turned severe during the observation period), and date of death (for patients who died during the observation period) $(6,7)$. We defined onset date as the date when any symptoms appeared (6); onset date was missing for patients who did not present any symptoms. The date when a patient's condition became severe was defined as the date when a patient met any of the following criteria: $i$ ) received mechanical ventilation, $i i$ ) received extracorporeal membrane oxygenation (ECMO), and iii) was admitted to the intensive care unit (ICU) (8).

\section{Definition of group}

We categorized COVID-19 patients in Osaka Prefecture who were confirmed as COVID-19 between February 1 and August 31 into two groups according to the date of the positive laboratory test result: patients who showed positive results in the laboratory test between February 1, 2020 and May 22, 2020 (the first surge) and patients who showed positive results in the laboratory test between May 23, 2020 to August 31, 2020 (the second surge). The Japanese government declared a state of emergency in Osaka Prefecture on April 7, 2020, and it lifted the state of emergency in Osaka Prefecture on May 21, 2020 (9). The end of the first surge was defined based on the following two reasons: $i$ ) patients whose samples were submitted for laboratory tests on May 21 (the end of the state of emergency) were reported on May 22; and ii) the number of newly diagnosed COVID-19 patients was zero on May 23 for the first time. The end of the second surge in this study was defined as the final day of the present study.

\section{Endpoints}

The outcome measures in this study were increased disease severity, and all-causes of mortality. All patients were followed-up for 30 days from the onset date.

\section{Statistical analyses}

The baseline characteristics of COVID-19 patients were compared between the first and the second surges. Sex, age group at the onset date $(0-59,60-69,70-79,80-89, \geq$ 90 years), and city of residence (Osaka City, other cities, unknown) were compared between the two groups using the chi-square test. Days from the onset date to the date when the patient showed positive results in the laboratory test (days to test positive) were summarized by interquartile range in each group, and they were compared between the two groups by applying the Wilcoxon rank sum test. We also categorized days to test positive by quartile, and analyzed the distribution of age groups according to the four categories.

We compared the severity of the condition of COVID-19 patients between the first and the second surges. Patients with missing information on the onset date or the date when the patient's condition became severe (for patients whose condition became severe during the observation period) were excluded from this analysis. The Kaplan-Meier method was applied to compare the cumulative probability of developing severe disease stratified by age group at the onset date (aged $\leq 69$ years or $\geq 70$ years), and the difference was compared between the first and the second surges by the log-rank test. The multivariable Cox proportionalhazards model was applied to compare disease severity between the two groups, where sex, age group at the onset date $(0-59,60-69,70-79,80-89, \geq 90$ years, unknown), city of residence (Osaka City, other cities, unknown), and days to test positive (categorized by quartile) were adjusted. We also stratified the study population according to age group at the onset date (aged $\leq 69$ years or $\geq 70$ years), and applied the multivariable Cox proportional-hazards model in each stratum.

We also compared the mortality of COVID-19 patients between the first and the second surges. Patients with missing information on the onset date or the date of death (for patients who died during the observation period) were excluded from this analysis. The Kaplan-Meier method was applied to compare cumulative mortality stratified by age group at the onset date (aged $\leq 69$ years or $\geq 70$ years), and the difference was compared between the first and the second surges by the log-rank test. The multivariable Cox proportional-hazards model was applied to compare mortality between the two groups. Variables adjusted in this model were the same as those adjusted in the multivariable Cox proportional-hazards model when we compared severity between the two groups. We also stratified the study population according to age group at the onset date (aged $\leq 69$ years or $\geq 70$ years), and applied the multivariable Cox proportional-hazards model in each stratum.

All analyses in the present study were conducted using STATA version 16.0 MP software (StataCorp LP). All tests were two-tailed, and we considered p-values < 0.05 as statistically significant. 


\section{Results}

A total of 8,541 patients were confirmed as COVID-19 positive between February 1 and August 31, 2020 in Osaka Prefecture, Japan. The first and second surges consisted of 1,780 and 6,761 patients, respectively. Figure 1 presents the number of laboratory tests and COVID-19 patients recorded per day in Osaka Prefecture, Japan. In the first surge, the daily number of newly diagnosed COVID-19 patients reached its peak on April 9 (92 cases per day). In the second surge, the daily number of newly diagnosed COVID-19 patients reached its peak on August 7 (255 cases per day).

Table 1 describes the characteristics of COVID-19 patients in the first and second surges. Although the difference in the distribution of sex was not statistically significant $(p=0.08)$, the proportion of male was higher in the second surge than in the first surge. The proportion of patients aged 0-59 years was higher in the second surge than in the first surge. The proportion of patients living in Osaka City was higher in the second surge than in the first surge. Days to test positive was significantly lower in the second surge than in the first surge $(p<0.001)$. Supplementary Table S1 (https:// www.globalhealthmedicine.com/site/supplementaldata. $h t m l ? I D=11)$ describes the distribution of age groups according to four categories (Q1-Q4) of days to test positive. The proportion of patients aged $\geq 70$ years was highest in Q1 (15.9\%), and lowest in Q3 (8.9\%).
Figure 2 shows the Kaplan-Meier curves for disease severity among patients aged $\leq 69$ years (Figure $2 \mathrm{~A}$ ) and $\geq 70$ years (Figure 2B); 7,122 patients were included in the analysis of severity. The log-rank test revealed that severity was significantly lower in the second surge than in the first surge in each stratum $(p$ $<0.001$ and $p=0.03$, for patients aged $\leq 69$ years and $\geq 70$ years, respectively). Table 2 describes the number of patients, number of events, and adjusted hazard ratios (HRs) with 95\% confidence intervals (CIs) by the multivariable Cox proportional-hazards model for severity. Severity was lower in the second surge than in the first surge (adjusted HR: 0.51, 95\% CI: 0.39$0.67)$. In the stratified analysis, severity was lower in the second surge than in the first surge among both patients aged $\leq 69$ years and those aged $\geq 70$ years (HR: 0.51 and 0.64 , for patients aged $\leq 69$ years and $\geq 70$ years, respectively). Supplementary Table S2 (https:// www.globalhealthmedicine.com/site/supplementaldata. html?ID=11) describes HRs and 95\% CIs for all the variables adjusted in the multivariable Cox regression analysis on severity. Higher severity was observed among male than among female (adjusted HR: 2.70, 95\% CI: 2.01-3.65). Significantly higher severity was observed among patients aged $\geq 60$ years compared with those aged $\leq 59$ years, except for that among patients aged $\geq 90$ years. Severity was similar regardless of the city of residence or days to test positive.

Figures 3(A) and 3(B) show the Kaplan-Meier
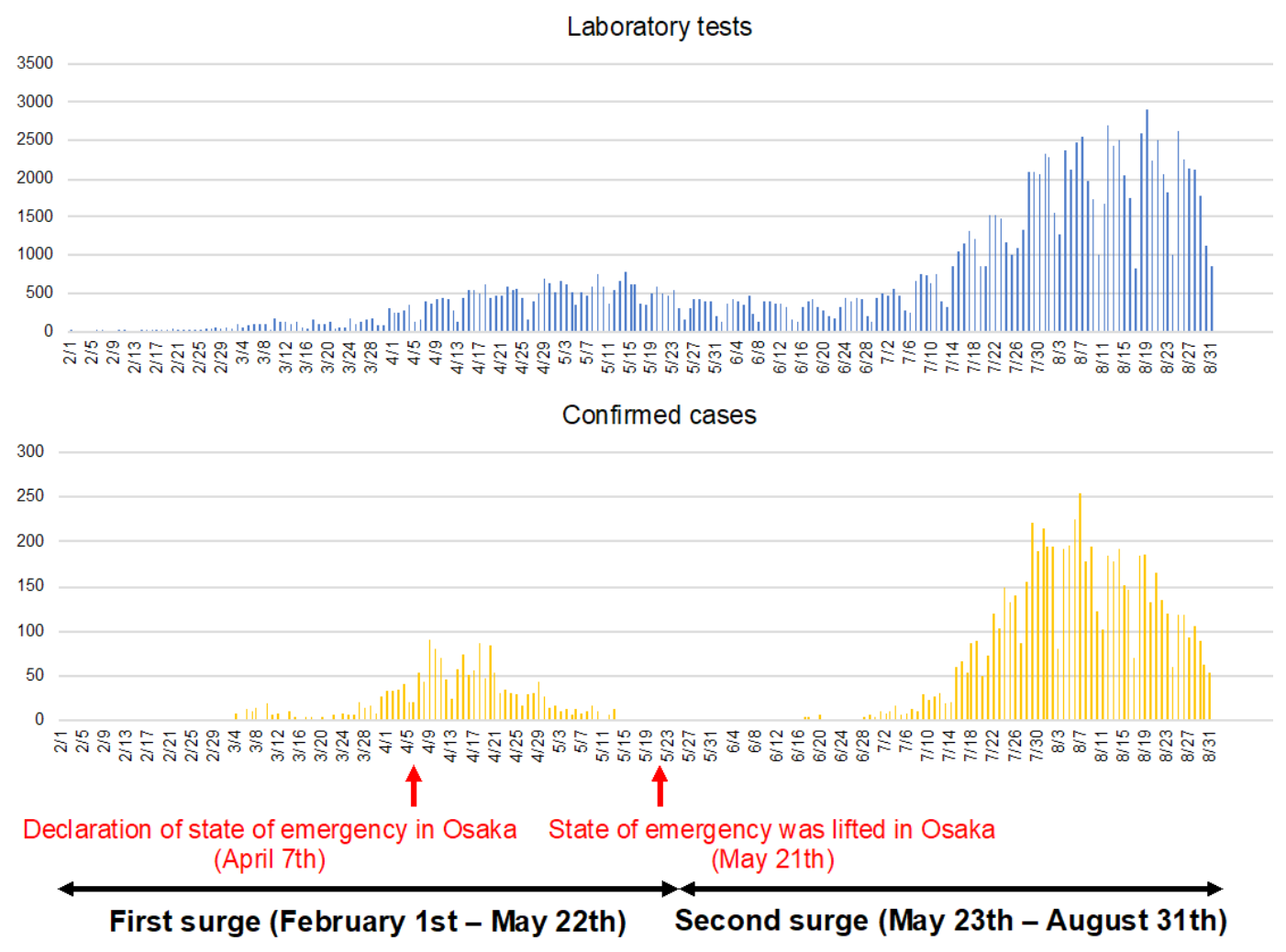

Figure 1. The number of laboratory tests and confirmed coronavirus disease (COVID-19) cases per day in Osaka Prefecture, Japan. 
Table 1. Characteristics of the study population according to surge

\begin{tabular}{|c|c|c|c|}
\hline \multirow[t]{2}{*}{ Variables } & $\begin{array}{c}\text { First surge } \\
\text { February } 1 \text { - May } 22\end{array}$ & $\begin{array}{c}\text { Second surge } \\
\text { May } 23 \text { - August } 31\end{array}$ & \multirow[t]{2}{*}{$p$ value } \\
\hline & $n=1,780$ & $n=6,761$ & \\
\hline \multicolumn{4}{|l|}{ Sex } \\
\hline Female & $804(45.2 \%)$ & $2,864(42.4 \%)$ & 0.08 \\
\hline Male & $976(54.8 \%)$ & $3,895(57.6 \%)$ & \\
\hline Unknown & $0(0.0 \%)$ & $2(0.0 \%)$ & \\
\hline \multicolumn{4}{|c|}{ Age group at the onset date } \\
\hline $0-59$ & $1,292(72.6 \%)$ & $5,531(81.8 \%)$ & $<0.001$ \\
\hline $60-69$ & $161(9.0 \%)$ & $419(6.2 \%)$ & \\
\hline $70-79$ & $176(9.9 \%)$ & $415(6.1 \%)$ & \\
\hline $80-89$ & $117(6.6 \%)$ & $307(4.5 \%)$ & \\
\hline$\geq 90$ & $33(1.9 \%)$ & $89(1.3 \%)$ & \\
\hline Unknown & $1(0.1 \%)$ & $0(0.0 \%)$ & \\
\hline \multicolumn{4}{|l|}{ City of residence } \\
\hline Osaka City & $737(41.4 \%)$ & $3,627(53.6 \%)$ & $<0.001$ \\
\hline Other cities & $876(49.2 \%)$ & $3,075(45.5 \%)$ & \\
\hline Unknown & $167(9.4 \%)$ & $59(0.9 \%)$ & \\
\hline Days to test positive & $7(5-10)$ & $6(4-8)$ & $<0.001$ \\
\hline
\end{tabular}

(A)

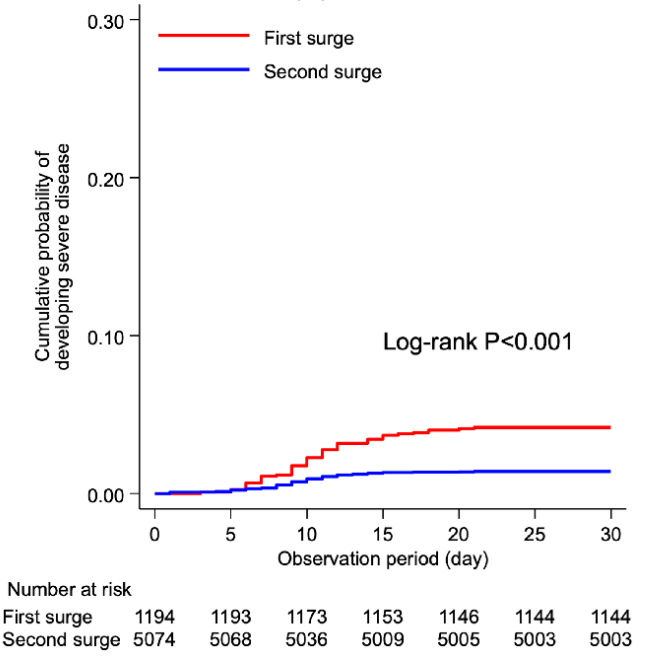

(B)

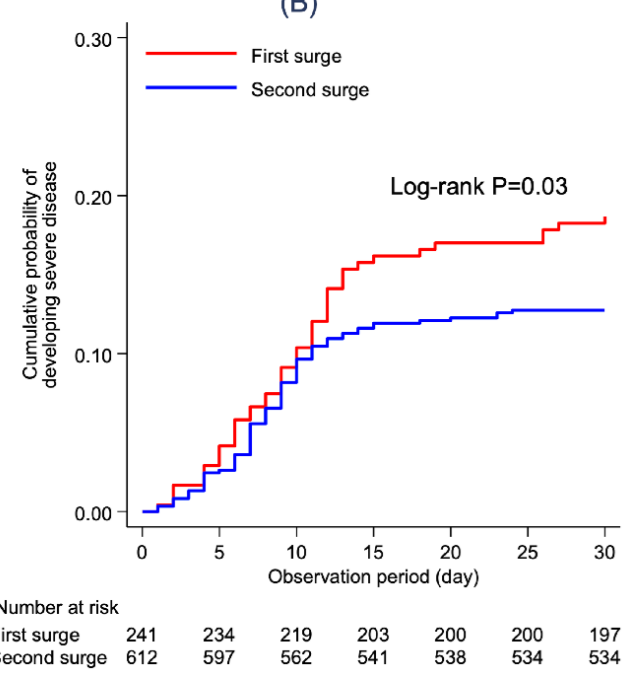

Figure 2. Kaplan-Meier curves for severity among patients aged $(A) \leq 69$ years and $(B) \geq 70$ years.

Table 2. The results of Cox regression analysis on severity

\begin{tabular}{lccc}
\hline Variables & Number of patients & Number of events & Adjusted HR (95\% CI) \\
\hline (i) All patients ${ }^{\dagger}$ & & & Ref \\
$\quad$ First surge (February 1 - May 22) & 1,436 & 95 & $0.51(0.39-0.67)$ \\
$\quad$ Second surge (May 23 - August 31) & 5,686 & 149 & Ref \\
(ii) Patients aged $\leq$ 69 years & & 50 & $0.51(0.35-0.74)$ \\
$\quad$ First surge (February 1 - May 22) & 1,194 & 71 & Ref \\
$\quad$ Second surge (May 23 - August 31) & 5,074 & 45 & $0.64(0.43-0.93)$ \\
(iii) Patients aged $\geq 70$ years & & 78 &
\end{tabular}

$\mathrm{HR}$, hazard ratio; $\mathrm{CI}$, confidence interval. 'In this model, sex, age group at the onset date (0-59, 60-69, 70-79, 80-89, $\geq 90$ years, unknown), city of residence (Osaka City, other cities, unknown), and days to test positive (categorized by quartile) were adjusted. 

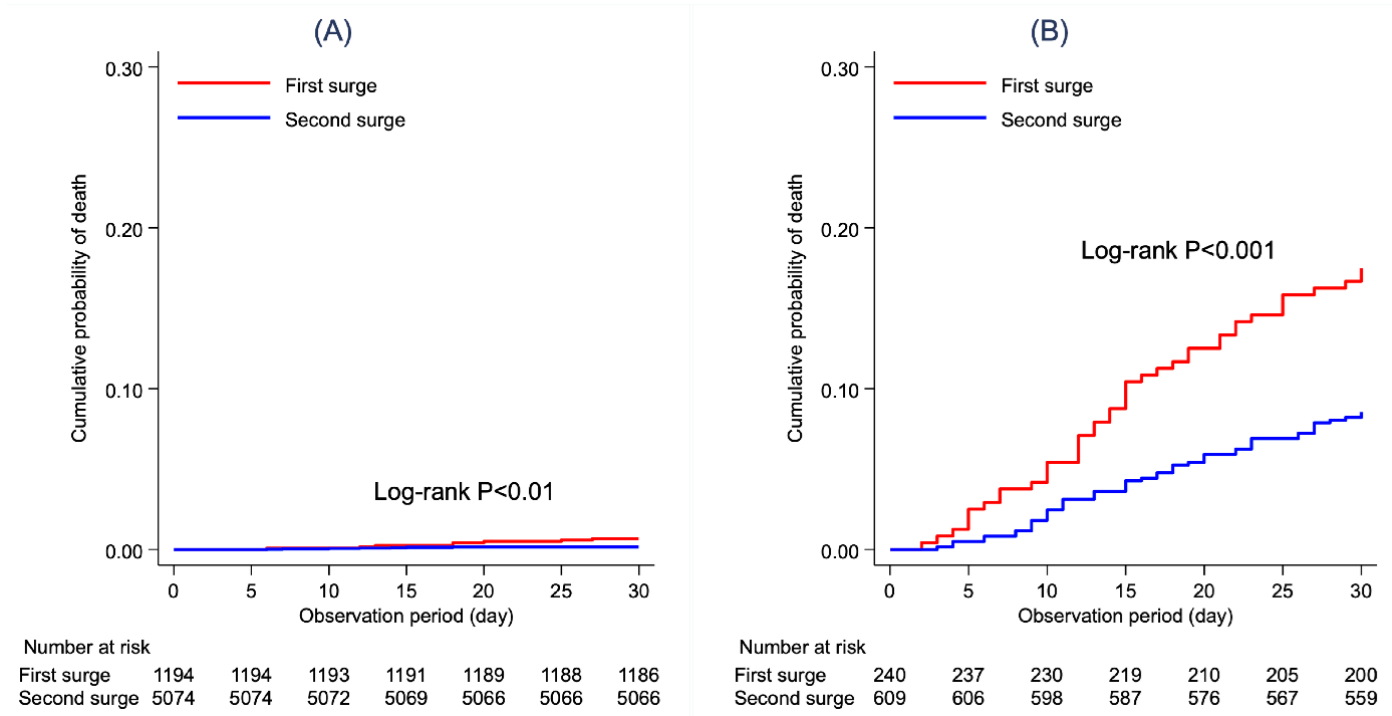

Figure 3. Kaplan-Meier curves for mortality among patients aged $(A) \leq 69$ years and $(B) \geq 70$ years.

Table 3. The results of Cox regression analysis on mortality

\begin{tabular}{lccc}
\hline Variables & Number of patients & Number of deaths & Adjusted HR (95\% CI) \\
\hline (i) All patients & & & $R e f$ \\
$\quad$ First surge (February 1 - May 22) & 1,435 & 50 & $0.37(0.25-0.56)$ \\
Second surge (May 23 - August 31) & 5,683 & 60 & $R e f$ \\
(ii) Patients aged $\leq$ 69 years & 1,194 & 8 & $0.36(0.13-1.00)$ \\
$\quad$ First surge (February 1 - May 22) & 5,074 & 8 & $R e f$ \\
Second surge (May 23 - August 31) & 240 & 42 & $0.38(0.25-0.59)$ \\
(iii) Patients aged $\geq 70$ years & 609 & 52 & \\
$\quad$ First surge (February 1 - May 22) & & & \\
Second surge (May 23 - August 31) &
\end{tabular}

HR, hazard ratio; CI, confidence interval. 'In this model, sex, age group at the onset date (0-59, 60-69, 70-79, 80-89, $\geq 90$ years, unknown), city of residence (Osaka City, other cities, unknown), and days to test positive (categorized by quartile) were adjusted.

curves for mortality among patients aged $\leq 69$ years and $\geq 70$ years, respectively. A total of 7,118 patients were included in the analysis of mortality. The log-rank test revealed that mortality was significantly lower in the second surge than in the first surge in each stratum $(p$ $<0.01$ and $p<0.001$, for patients aged $\leq 69$ years and $\geq 70$ years, respectively). Table 3 presents the number of patients, number of events, and adjusted HR with 95\% CI by the multivariable Cox proportional-hazards model for mortality. Mortality was lower in the second surge than in the first surge (adjusted HR: $0.37,95 \%$ CI: $0.25-0.56)$. In the stratified analysis, mortality was lower in the second surge than in the first surge among both patients aged $\leq 69$ years and those aged $\geq 70$ years (HR: 0.36 and 0.38 , for patients aged $\leq 69$ years and $\geq$ 70 years, respectively). Supplementary Table S3 (https:// www.globalhealthmedicine.com/site/supplementaldata. html?ID=11) describes HRs and 95\% CIs for all the variables adjusted in the multivariable Cox regression analysis on mortality. Higher mortality was observed among male than among female (adjusted HR: 1.99, 95\%
CI: 1.32-3.02). Higher age was significantly associated with higher mortality. Mortality was similar regardless of the city of residence. Lower mortality was observed in the highest and the second highest quartiles of days to test positive compared with the lowest quartile.

\section{Discussion}

The present study targeted 8,541 COVID-19 patients between February 1 and August 31, 2020 in Osaka Prefecture, Japan, and compared the characteristics, severity, and mortality of patients between the first and the second surges of infections. In the second surge, the age group at the onset date was younger, the number of days to a positive test was lower, and the severity and mortality were lower.

In this study, both severity and mortality were lower in the second surge than in the first surge, even when we adjusted for available confounding factors. We speculate that this result could be explained in part by the following four factors: $i$ ) advancement in treatments for 
COVID-19; ii) behavioral changes and awareness among citizens at higher risk of developing severe disease; iii) lower number of clusters at nursing homes; and $i v$ ) attenuation of SARS-CoV-2.

As of September 30, 2020, there are few drug therapies and no vaccine for COVID-19. However, the treatments for COVID-19 patients have improved over time. Several drug therapies have been approved in Japan. Remdesivir, a nucleotide analog prodrug that inhibits viral RNA polymerases (10), was fast-track approved in Japan by the Ministry of Health, Labour, and Welfare on May 7, 2020 (11). Dexamethasone, a steroid drug, was also fast-track approved in Japan by the Ministry of Health, Labour, and Welfare in July 2020 (12). Clinical trials for other potential drug therapies including Favipiravir, are also in progress (11). Intensive care for patients with severe COVID-19, including mechanical ventilation and extracorporeal membrane oxygenation (ECMO) (11), has also been improved. Based on the results of clinical studies on COVID-19 patients and improvements in treatments for COVID-19, a guide for the clinical management of patients with COVID-19 for front-line healthcare workers is now available online (11). Several scientific societies have also published guidelines for treatment of COVID-19 patients (13), and evidence on clinical care of COVID-19 patients has been accumulated. We speculate that improvements in treatments for COVID-19 patients may have resulted in lower severity and mortality in the second surge than in the first surge.

Behavioral changes and awareness over time among Japanese citizens at higher risk of developing severe disease may also have resulted in lower severity and mortality in the second surge. The three Cs, namely closed spaces, crowded places, and close-contact settings (4), have been gradually recognized as important for the spread of the COVID-19 pandemic, and new lifestyles termed as the "new normal" lifestyles including maintaining social distancing and using hand sanitizers have gradually become established among Japanese citizens. In particular, a previous study (14) reported that such behavioral changes and awareness were more frequently observed among elderly people compared to young people. Elderly people are reported to be at higher risk of developing severe disease and that of death among COVID-19 patients (6,15-18). Therefore, we speculate that behavioral changes and awareness among citizens at higher risk of developing severe disease may have led to the lower proportion of these citizens among COVID-19 patients, which may have resulted in lower severity and mortality in the second surge.

Fewer clusters at nursing homes in the second surge than in the first surge would also be associated with lower severity and mortality in the second surge. Previous studies $(19,20)$ reported that mortality from COVID-19 was high among residents of nursing homes. Such residents are generally frail (19), and they are speculated to be at higher risk for developing severe disease compared with elderly citizens who do not live in nursing homes. We speculate that there were fewer clusters at nursing homes in the second surge based on experiences in the first surge, which would have resulted in lower severity and mortality.

Lower severity and mortality could also be explained in part by the attenuation of SARS-CoV-2. Previous in vitro studies on SARS-CoV, which was responsible for the SARS pandemic, showed that deletion in SARS$\mathrm{CoV}$ genome led to lower efficiency of SARS-CoV replication, which could have resulted in milder clinical illness $(21,22)$. Such a mutation was also reported in SARS-CoV-2, and was reported to be associated with a milder infection (23). Although the effect of mutation on the COVID-19 pandemic is yet to be elucidated, mutation in the SARS-CoV-2 genome may have resulted in lower severity and mortality in the second surge. Further genome and virus studies would be needed to elucidate the attenuation of SARS-CoV-2 in Japan.

In our study, the proportion of elderly patients was lower in the second surge than in the first surge. This result could also be explained in part by two reasons: $i$ ) behavioral changes and awareness among elderly people who were at higher risk of developing severe disease (14); and ii) an increased number of laboratory tests for patients who did not present any symptoms in the second surge, especially among younger citizens.

Days to test positive were also significantly lower in the second surge than in the first surge. As we have described in a previous report (6), the laboratory testing system for COVID-19 was not widely adopted in Japan in the first surge. Therefore, it is speculated that it took longer for COVID-19 patients to be detected as positive in the first surge. However, by May 2020, the laboratory testing system became more available. Although the maximum number of daily tests was below 1,000 in the first surge, this number was approximately 3,000 in the second surge, as shown in Figure 1. It is speculated that widespread use of laboratory testing systems led to earlier detection of COVID-19 patients. However, fewer days to test positive was not associated with lower severity and mortality, as shown in Supplementary Tables S2 and S3 (https://www.globalhealthmedicine. com/site/supplementaldata.html? ID=11). In addition, higher mortality was observed among patients in the fewest category (Q1) of days to test positive compared with the highest (Q4) and second highest (Q3) categories. The proportion of patients aged $\geq 70$ years was highest in Q1 (Supplementary Table S1, https:// www.globalhealthmedicine.com/site/supplementaldata. html?ID=11). In Osaka Prefecture, when elderly facility residents and clusters among elderly patients were suspected to be infected with COVID-19, laboratory tests for such residents were conducted as soon as possible. We speculate that such residents, who were at higher risk of developing severe disease, underwent laboratory tests 
rapidly; even though, some proportion of these residents developed severe disease or died subsequently.

Previous studies have compared the characteristics of COVID-19 patients between the first and the second surges. A previous study conducted in Texas in the United States (5) compared the characteristics and outcomes of COVID-19 patients between surge 1 (March 13, 2020 to May 15, 2020) and surge 2 (May 16, 2020 to July 7, 2020), and found that patients in surge 2 were younger and had lower in-hospital mortality in surge 2 (5), both of which were consistent with our findings. A previous study (24) in Wuhan categorized patients into three groups: group A (January 21 to January 25), group B (January 26 to January 31 ), and group C (February 1 to February 10), and found that all-causes of mortality significantly decreased over time (24); this was also consistent with our findings. A recent study in Japan (25) compared characteristics and severity between the first and second COVID-19 waves in Japan, and concluded that the proportion of severe cases on admission was lower in the second wave. Further studies are needed to examine changes in the characteristics of COVID-19 patients over time. Especially, from November 2020, since Japan is in the middle of the third surge of infections (4). In Osaka Prefecture, as of December 5, 2020, the daily number of newly diagnosed COVID-19 patients was the highest ever on November 22, 2020 (490 cases per day) (7). Future studies will be needed to compare severity and mortality between the first, second, and third surge of infections.

There were several limitations to the present study. First, we did not have information on patient age (not age group) at the onset. We could not adjust for age in the multivariable analysis. Instead, the age group at the onset date was adjusted, which could have resulted in residual confounding. Second, we could not compare causes of death among COVID-19 patients between the first and the second surges, because this information was not publicly available (6). Third, we could not include patients with missing information on the onset date/date when the patient's condition became severe/date of death in the survival analyses. Finally, information on patient characteristics such as medical history, comorbidities, inhospital treatments, and health status was not publicly available, and we could not adjust for these factors in the multivariable analysis (6).

In conclusion, in Osaka Prefecture, Japan, severity and mortality were significantly lower in the second surge than in the first surge, among COVID-19 patients. It was also concluded that the number of days to test positive was lower in the second surge than in the first surge. Further studies are needed to analyze the severity and mortality in COVID-19 patients. Although the results of this study cannot explain why severity and mortality was lower in the second surge, greater advancement in treatments for COVID-19, behavioral changes and awareness among citizens, and effective measures to prevent clusters at nursing homes are needed for disease control. In addition, intensive and careful treatments are needed for elderly and male patients, who are at higher risk.

\section{Acknowledgements}

Takeuchi T was supported by the Osaka University Medical Doctor Scientist Training Program. We thank our colleagues from Osaka University Center of Medical Data Science and Advanced Clinical Epidemiology Investigator's Research Project for providing insight and expertise for our research.

\section{Funding: None.}

Conflict of Interest: The authors have no conflicts of interest to disclose.

\section{References}

1. Huang C, Wang Y, Li X, et al. Clinical features of patients infected with 2019 novel coronavirus in Wuhan, China. Lancet. 2020; 395:497-506.

2. Chen N, Zhou M, Dong X, Qu J, Gong F, Han Y, Qiu Y, Wang J, Liu Y, Wei Y, Xia J, Yu T, Zhang X, Zhang L. Epidemiological and clinical characteristics of 99 cases of 2019 novel coronavirus pneumonia in Wuhan, China: a descriptive study. Lancet. 2020; 395:507-513.

3. Lai CC, Wang CY, Wang YH, Hsueh SC, Ko WC, Hsueh PR. Global epidemiology of coronavirus disease 2019 (COVID-19): disease incidence, daily cumulative index, mortality, and their association with country healthcare resources and economic status. Int J Antimicrob Agents. 2020; 55:105946.

4. Ministry of Health, Labour and Welfare of Japan. The Statistics of Patients with COVID-19. https://www.mhlw. go.jp/stf/newpage_13292.html (accessed October 10, 2020) (in Japanese).

5. Vahidy FS, Drews AL, Masud FN, Schwartz RL, Askary BB, Boom ML, Phillips RA. Characteristics and Outcomes of COVID-19 Patients During Initial Peak and Resurgence in the Houston Metropolitan Area. JAMA. 2020; 324:998-1000.

6. Takeuchi T, Imanaka T, Katayama Y, Kitamura T, Sobue T, Shimazu T. Profile of patients with novel coronavirus disease 2019 (COVID-19) in Osaka Prefecture, Japan: a population-based descriptive study. J Clin Med. 2020; 9:2925.

7. Osaka Prefectural Government. The Press Releases and Documents about COVID-19 Patients. http://www.pref. osaka.lg.jp/iryo/osakakansensho/corona.html (accessed October 10, 2020) (in Japanese).

8. Ministry of Health, Labour and Welfare of Japan. Request for survey report on medical treatment status of patients with new coronavirus infection from the Ministry of Health, Labour and Welfare on April 26, 2020. https:// www.mhlw.go.jp/content/000625945.pdf (accessed October 12, 2020) (in Japanese).

9. Prime Minister of Japan and His Cabinet. The official homepage of Prime Minister of Japan and His Cabinet. https://japan.kantei.go.jp/(accessed October 10, 2020). 
10. Grein J, Ohmagari N, Shin D, et al. Compassionate use of remdesivir for patients with severe Covid-19. N Engl J Med. 2020; 382:2327-2336.

11. Ministry of Health, Labour and Welfare of Japan. Clinical Management of Patients with COVID-19: A guide for front-line healthcare workers, version 3. https://www. mhlw.go.jp/content/000670444.pdf (accessed October 10, 2020) (in Japanese).

12. Prime Minister of Japan and His Cabinet. The official homepage of Prime Minister's Office of Japan. https:// www.kantei.go.jp (accessed October 10, 2020) (in Japanese).

13. The Japanese Society of Intensive Care Medicine. The special version of the Japanese Clinical Practice Guidelines for Management of Sepsis and Septic Shock 2020 (J-SSCG2020): Rapid/Living recommendations for drug therapies of COVID-19, ver.2.0.0_2020.10.14. https://www.jaam.jp/info/2020/files/info-20201014_1.pdf (accessed October 17, 2020) (in Japanese).

14. Muto K, Yamamoto I, Nagasu M, Tanaka M, Wada K. Japanese citizens' behavioral changes and preparedness against COVID-19: an online survey during the early phase of the pandemic. PLoS One. 2020; 15:e234292.

15. Suleyman G, Fadel RA, Malette KM, et al. Clinical characteristics and morbidity associated with coronavirus disease 2019 in a series of patients in metropolitan Detroit. JAMA Netw Open. 2020; 3:e2012270.

16. Munblit D, Nekliudov NA, Bugaeva P, et al. StopCOVID cohort: an observational study of 3,480 patients admitted to the Sechenov University hospital network in Moscow city for suspected COVID-19 infection. Clin Infect Dis. 2020; ciaa1535.

17. Wei C, Liu Y, Liu Y, Zhang K, Su D, Zhong M, Meng X. Clinical characteristics and manifestations in older patients with COVID-19. BMC Geriatr. 2020; 20:395.

18. Hirayama A, Masui J, Murayama A, Fujita S, Okamoto J, Tanaka J, Hirayama T, Ohara T, Hoffmann EN, Zhang J, Kawachi H, Okuno H, Hamaguchi S, Tomono K, Asada $\mathrm{R}$. The characteristics and clinical course of patients with COVID-19 who received invasive mechanical ventilation in Osaka, Japan. Int J Infect Dis. 2020; 102:282-284.
19. Kittang BR, Hofacker SV, Solheim SP, Krüger K, Løland KK, Jansen K. Outbreak of COVID-19 at three nursing homes in Bergen. Tidsskr Nor Laegeforen. 2020; 140. doi: 10.4045/tidsskr.20.0405.

20. Brouns SH, Brüggemann R, Linkens AEMJH, Magdelijns FJ, Joosten H, Heijnen R, Ten Cate-Hoek AJ, Schols JMGA, Ten Cate H, Spaetgens B. Mortality and the use of antithrombotic therapies among nursing home residents with COVID-19. J Am Geriatr Soc. 2020; 68:1647-1652.

21. Muth D, Corman VM, Roth $\mathrm{H}$, et al. Attenuation of replication by a 29 nucleotide deletion in SARScoronavirus acquired during the early stages of human-tohuman transmission. Sci Rep. 2018; 8:15177.

22. Cui J, Li F, Shi ZL. Origin and evolution of pathogenic coronaviruses. Nat Rev Microbiol. 2019; 17:181-192.

23. Young BE, Fong SW, Chan $\mathrm{YH}$, et al. Effects of a major deletion in the SARS-CoV-2 genome on the severity of infection and the inflammatory response: an observational cohort study. Lancet. 2020; 396:603-611.

24. Wang M, Zhang J, Ye D, et al. Time-dependent changes in the clinical characteristics and prognosis of hospitalized COVID-19 patients in Wuhan, China: a retrospective study. Clin Chim Acta. 2020; 510:220-227.

25. Saito S, Asai Y, Matsunaga N, Hayakawa K, Terada M, Ohtsu H, Tsuzuki S, Ohmagari N. First and second COVID-19 waves in Japan: a comparison of disease severity and characteristics. J Infect. 2020:S01634453(20)30693-9.

Received October 28, 2020; Revised December 10, 2020; Accepted December 15, 2020.

Released online in J-STAGE as advance publication December 30, 2020.

*Address correspondence to:

Tetsuhisa Kitamura, Department of Social Medicine, Osaka University Graduate School of Medicine, 2-2 Yamadaoka, Suita, Osaka 565-0871, Japan.

E-mail: lucky_unatan@yahoo.co.jp 